\title{
Preface for the special issue "Biomembranes"
}

\author{
Mohamed Laradji ${ }^{2}$ P. B. Sunil Kumar ${ }^{1}$
}

Published online: 27 June 2016

(C) Indian Institute of Technology Madras 2016

Biomembranes are fascinating self-assemblies of lipids, which act as a physical barrier between the inner and outer environment of cells, compartmentalizes the cell, mediates cell signaling and support various proteins that are crucial for a multitude of cellular functions. A large amount of experimental, theoretical and computational studies have been devoted to understanding the biophysical properties of biomembranes. The present special issue includes a collection of seven articles, from leaders in the field, that focus on few structural and thermodynamic aspects of lipid membranes, using a variety of computational and theoretical methods.

This special issue begins with an investigation of factors that control the morphology of membranes through an article, by Ramakrshnan, Tourdot and Radhakrishnan. This article is focused on a set of different theoretical methods for investigating the morphological transitions in lipid membranes as a result of curvature-remodeling proteins or as a result of external forces. Electrostatics plays an important role in determining membrane morphology. This is the subject of the article by Bossa, Brown, Bohinc and May. Here, they present a theory calculating the contribution of electrostatics to the line tension between coexisting domains in multicomponent lipid membranes. This is followed by an article from Brodbek and Schmid, which describes a mean field theory for an investigation of the effect of the interplay between composition and local curvature of lipid membranes on their phase behavior. An outcome of this work is that external tension on the lipid membrane can have an effect on the organization of lipid rafts. Continuing on the theme of morphology of membranes, Ipsen's article presents a theoretical procedure for the representation of Gaussian models for lipid membranes, as described by triangulated surfaces, with in-plane directional degrees of freedom.

While the above set of articles are focused on the macroscopic structure of membranes the next two articles focus on mesocale descriptions of membranes. Vanherpe, Kanari, Atenekeng, Palacios, and Shillcock present an approach for the in situ synthesis of polydisperse lipid membranes for coarse-grained simulations with a particular application to a dissipative particle dynamics model for multicomponent membranes. Parisio, Ferrarini and Sperotto's article reviews the current numerical approaches to investigate flip-flops of various phospholipids and sterols.

The final article explores other forms if lipid assemblies. Penic, Perutkova, Fosnaric and Iglic presented two Monte Carlo approaches for investigating lamellar and inverted hexagonal phases of lipid self-assemblies.

We hope that the readers will find these articles to be enjoyable, useful and enlightening.
$凶$ P. B. Sunil Kumar

sunil@iitm.ac.in

Mohamed Laradji

mlaradji@memphis.edu

1 Chennai, India

2 Memphis, TN, USA 